\title{
CULTURA, EDUCACIÓN Y CUIDADOS EN LA ATENCIÓN AL PACIENTE OSTOMIZADO
}

\author{
Rita PULIDO JUSTICIA* \\ Judit SÁNCHEZ GARCÍA ** \\ Aurora BARAZA SAZ *
}

*Supervisoras de Enfermería. Unidad de Docencia. Hospital de G. C. Dr. Negrín

**Profesora Asociada a tiempo completo. Escuela Universitaria de Enfermería de la U.L.P.G.C.

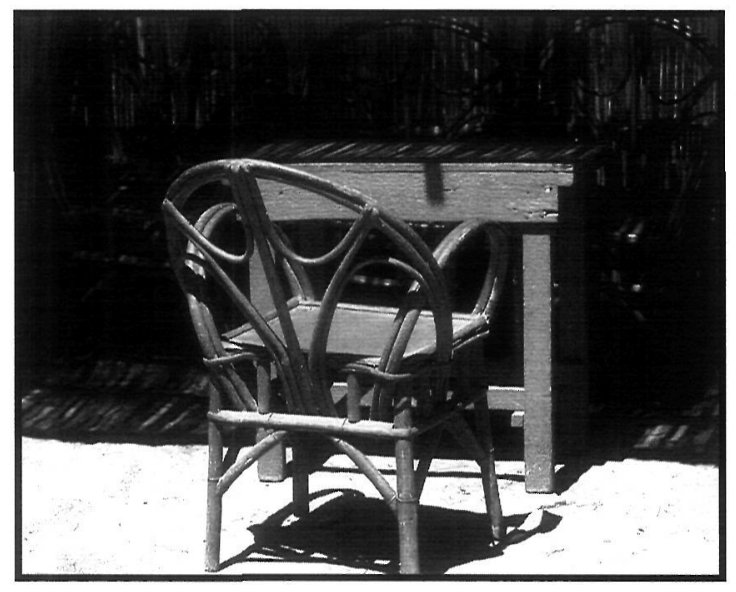

CULTURE, EDUCATION AND CARE OF THE PATIENT WITH AN OSTOMY



dequate Nursing care demands anthropological knowledge of the people who are to be cared for. Patients with an ostomy experience changes, alterations and disturbances by their new situation at all levels: physical, psychological, social and cultural. This knowledge provides us with the basis for flexible, adapted and individualised care in order to prevent, minimise or sort out the problems that may arise.

Our work is based upon Virginia Henderson's holistic and humanistic perspective and nurses' autonomy and authority to deal with the patient's trouble and satisfactory and independently fulfill his/her basic needs.

Literature has been reviewed in search of the ways in which culture and education determine acceptance and independence of the patient with an ostomy.

\section{RESUMEN}

$\mathrm{P}$ ara la adecuada aplicación de los cuidados enfermeros es necesario el conocimiento antropológico de las personas a las que van dirigidos. Concretando en las personas ostomizadas debemos evidenciar los cambios, modificaciones y trastornos producidos por la nueva situación (ser portador de un estoma), a todos los niveles: físico, psicológico, social y cultural. Estos conocimientos nos proporcionan la base para proporcionar unos cuidados flexibles, adaptados e individualizados dirigidos a prevenir, disminuir o solucionar los problemas que puedan presentar.

Nos basamos en la visión holística y humanista de Virginia Henderson y en al autoridad y autonomía que tenemos las enfermeras para tratar las alteraciones que presenta la persona para cubrir de forma satisfactoria e independiente sus necesidades básicas.

Hemos realizado un revisión bibliográfica para conocer como la cultura y la educación afectan a la aceptación y al logro de la independencia de la persona ostomizada.

\section{INTRODUCCIÓN}

Tal y como observa Collière en su artículo "Utilización de la antropología para abordar situaciones de cuidados" toda situación de cuidados, es en sí una situación antropológica en la que el cuidador desconoce todo lo que es propio del paciente: sus hábitos, sus creencias, su forma de llevar la enfermedad. Por lo que se hace necesaria una aproximación antropológica, el cuidador ha de acercarse a la gente, ha de reencontrarse con las personas, ha de identificar todo aquello a lo que la persona 
que requiere cuidados le concede importancia. Necesita ver y escuchar, para descubrir y aprender.

En la línea descrita por Collière, pretendemos desarrollar el presente trabajo, con la intención de evidenciar los cambios, las modificaciones, los trastornos que presentan las personas portadoras de un estoma (traqueostomía, ileostomía, colostomía y urostomía), a nivel psicológico, social y cultural. Así como la atención y cuidados que los profesionales enfermeros podemos realizar para prevenirlos, disminuirlos y/o solucionarlos.

La aproximación antropológica tiene como finalidad captar como se estructura una situación sociocultural y extraer los lazos de significación. Aunque va a ser la enfermedad quien oriente aisladamente y de forma predominante el proceso de los cuidados.

Es necesario apreciar la dinámica de las interrelaciones que tienen lugar en todas las situaciones de cuidados. El conocimiento de la historia de vida del paciente, el acontecimiento y las preocupaciones que suscita la enfermedad, las afectaciones o perturbaciones funcionales y el entorno, van a permitir la elaboración de procesos de cuidados adaptados y flexibles, dirigidos a la persona cuidada.

A la persona que va a ser portadora de un estoma, en principio la consideramos enferma, ya que posee una alteración fisiopatológica y se enfrenta a una nueva situación en la que aparecerán nuevas necesidades que tendrá que cubrir. Dependiendo de su respuesta al proceso, de su capacidad de adaptación y superación, de la respuesta de su entorno familiar, social y laboral, y de la actuación de los profesionales sanitarios, puede o no ser considerada como enferma.

La conducta de enfermedad, se refiere a la atención concedida por el ostomizado a la sintomatología física, a la definición y al significado atribuidos al estoma y a la conducta de búsqueda de apoyo a través de los diversos servicios sanitarios.

Nuestra actuación profesional, en el presente trabajo, se sustenta en la concepción de Virginia Henderson y su visión holística y humanística de la persona, estableciéndose el vínculo biológico-cultural entre las catorce necesidades básicas que expone en su modelo.

Virginia Henderson define la profesión enfermera y dice, la función de la enfermería es asistir al individuo, enfermo o no, en la realización de esas actividades que contribuyen a la salud o su recuperación (o una muerte digna) y que él llevaría a cabo si tuviera la fuerza, la voluntad o el conocimiento necesarios. Y hacer estos de tal manera que le ayude a adquirir independencia lo más rápidamente posible.

Henderson nos dice que las enfermeras debemos y estamos autorizadas para actuar cuando la persona tiene alteradas sus capacidades para cubrir sus necesidades básicas, y define catorce necesidades presentes en todos los individuos. Esta visión de nuestra profesión es ampliamente aceptada en nuestro contexto, con pequeñas matizaciones derivadas del tipo de formación y de los recursos disponibles.

Vamos a ver, de forma somera, como estas 14 necesidades pueden verse alteradas en las personas ostomizadas. No vamos a profundizar en el tema, sino a poner de manifiesto la repercusión que un estoma puede tener en el conjunto de la persona.

1. Necesidad de respirar normalmente. En el paciente traqueostomizado se anula la función de la vía aérea superficial, creando una abertura artificial que permite la respiración pero que tiene sus deficiencias a nivel de humidificación, calentamiento, prevención de infecciones...

2. Comer y beber adecuadamente. El paciente traqueostomizado puede tener alterado el olfato y la deglución. Los pacientes con ostomías intestinales y urinarias deben individualizar su alimentación en función de la porción de intestino abocada y de las características del efluyente, en ocasiones es necesario modificar algunos hábitos.

3. Eliminar por todas las vías corporales. En función del tipo de estoma la eliminación no se realiza por la vía natural. En el traqueostomizado se evidencian las secreciones mucosas, en los ileostomizados y urostomizados está la presencia permanente de un reservorio artificial y en los colostomizados la necesidad de actuar para controlar las deposiciones.

4. Moverse y mantener posturas adecuadas. Esta necesidad está más alterada en el periodo de 
recuperación postquirúrgica. Posteriormente $\mathrm{y}$, si la elección del lugar del estoma y del tipo de dispositivo ha sido correcta, no tiene por qué haber alteración.

5. Dormir y descansar. En los traqueostomizados puede ser necesario dormir un poco incorporados y, los portadores de ostomías de eliminación, en los primeros meses pueden ver interferido el descanso por miedo a escapes o fugas.

6. Escoger ropa adecuada: vestirse y desvestirse. Los traqueostomizados por cuestión estética, para calentar el aire y para evitar manchar por las secreciones, utilizan prendas que tapan o disimulan el estoma. Los portadores de ostomías de eliminación modifican su forma de vestir para disimular el dispositivo colector.

\section{Mantener la temperatura corporal dentro} de los límites normales. La temperatura puede verse alterada en estos pacientes por posibles problemas de infección (mayor riesgo en los pacientes traqueostomizados) y de deshidratación por alteraciones en la eliminación (diarreas importantes) o sobrecolonización bacteriana.

8. Mantener la higiene corporal y la integridad de la piel. El todos los casos el problema viene derivado por el contacto del efluyente con la piel y por los materiales que se usan. Los problemas varían en función del contenido eliminado.

\section{Evitar peligros ambientales y evitar lesio-} nar a otras personas. Por la pérdida del control sobre la eliminación, el ostomizado puede tener miedo a encontrarse con situaciones embarazosas al estar en contacto con otras personas.

\section{Comunicarse con los demás expresando} emociones, necesidades, temores u opiniones. En el traqueostomizado hay una alteración real en la comunicación verbal que puede solventarse o no con el tiempo. En esta necesidad se incluye la comunicación afectiva y sexual, alteradas en la mayoría de los casos por temor al rechazo, rechazos reales y/o por alteraciones fisiológicas secundarias a la propia enfermedad o a la intervención. La vida social también se ve afectada por las mismas causas y la perdida de control en la eliminación. La expresión de emociones, opiniones y sentimientos lleva implícito evidenciar el problema y, en la mayoría de los casos, la tendencia es a ocultarlo.

11. Rendir culto según sus propias creencias. Tiene más importancia en los estomas intestinales y urinarios, y depende de los valores, normas y rituales impuestos por la $\mathrm{Fe}$ que se profese.

\section{Trabajar de tal forma que su labor tenga} un sentido de realización personal. La vida laboral tiene una faceta de realización personal y otra de relación social impuesta. Algunos ostomizados por el tipo de trabajo (esfuerzos físicos importantes, partículas en suspensión...) y otros por la falta de condiciones higiénicas deben modificar su actividad profesional.

\section{Jugar y participar en actividades recrea-} tivas. La alteración dependerá de las preferencias de la persona, en principio no existen restricciones como tales, pero debemos tener presente que esta necesidad está íntimamente relacionada con las relaciones sociales.

14. Aprender, descubrir o satisfacer la curiosidad que conduce a un desarrollo normal y a utilizar los medios sanitarios existentes. En principio todo ostomizado tiene un déficit de conocimientos ante la nueva situación.

\section{MATERIAL Y MÉTODOS}

Para la realización del trabajo se han revisado artículos publicados, en las Revistas: Rol, Enfermería Científica, Enfermería Clínica, Española de Enfermedades Digestivas y del CORCE, relacionados con los aspectos psicológicos, sociales, culturales y educacionales, en las personas ostomizadas.

Dentro de nuestro contexto, se analizan las medidas correctoras que los profesionales sanitarios, mayoritariamente enfermeras, estomaterapeutas y en algunos casos el conjunto de profesionales del equipo interdisciplinar, han aplicado u observado como necesarias, para solucionar el problema.

Para la búsqueda bibliográfica, utilizamos los 
siguientes recursos:

1. Búsqueda bibliográfica en línea sobre las siguientes bases de datos sanitarias accesibles en Internet:

-Medline:

http://www.ncbi.nlm.nih.gov/entrez/query.fcgi -Cuiden:

http://www.doc6.es/index/

-BDIE grupo INVESTEN:

http://www.isciii.es/investen/bdie/

2. Identificados los artículos de interés, procedimos a su localización, consultando en:

- Biblioteca del Hospital de Gran Canaria Dr. Negrín.

- Biblioteca de la Universidad de Las Palmas de Gran Canaria.

3. En las bibliotecas, además de los artículos, se buscaron libros que destacasen la influencia de la cultura-religión en el proceso salud-enfermedad.

Los datos que se analizaron, fueron agrupados teniendo en cuenta los siguientes aspectos:

- Alteraciones a nivel psicológico, familiar, social y laboral.

- Información, educación sanitaria y demanda de atención sanitaria.

- Capacidad de autocuidado.

- Valores religiosos en el proceso salud-enfermedad.

\section{RESULTADOS}

Siguiendo la agrupación anteriormente descrita, clasificamos los resultados obtenidos:

Alteraciones a nivel psicológico, familiar, social y laboral. En los artículos revisados, relacionados con el tema, encontramos dentro de las alteraciones a nivel psicológico, que los que se presentan con mayor frecuencia son los problemas emocionales, estados de ansiedad y depresión, seguidos de rechazo al estoma y resignación frente al mismo. Un reducido grupo acepta su nueva situación y finalmente otro pequeño grupo demuestra una actividad ritualista en torno a los cuidados. Finalmente detectamos la presencia de ansiedad y angustia permanente como alteración psicológica, aunque no se da normalmente.

La mayor parte de los pacientes ostomizados tenían un buen apoyo familiar, aunque aproximadamente la mitad de ellos tenían influencias negativas en su relación de pareja, siendo más frecuente la modificación de relaciones sexuales a causa del estoma en los hombres que en las mujeres.

Las alteraciones sociales que detectamos en nuestra revisión son la reducción de relaciones y capacidad de hacer amigos, manifestada en algunos casos como alteración del ocio.

Finalmente en este apartado, confirmamos que gran parte de los pacientes ostomizados presentaban problemas o interferencias en el trabajo.

Información, educación sanitaria y demanda de atención sanitaria. En la bibliografía consultada encontramos que muchos pacientes, no todos, referían no haber recibido ningún tipo de información por parte del personal de enfermería. Los que habían sido informados consideraban insuficiente la información y prácticamente la totalidad desconocía lo que era un estoma, más allá del orificio que observaban en la pared abdominal. Pensaban que hubiese sido beneficiosa una información adicional después de la intervención.

De los pacientes a los que se les informó de las rutinas preoperatorias, sin profundizar en el significado del estoma, una parte estaban satisfechos en el preoperatorio y otra en el postoperatorio; Frente al $100 \%$ de los pacientes a los que se les realizó un programa de educación sanitaria estructurado, estos mostraban un alto grado de satisfacción en todo el proceso.

Casi todas las personas ostomizadas demandaban atención sanitaria en su centro hospitalario (presencia física). Las consultas telefónicas realizadas por los familiares, en los casos revisados, era el medio de comunicación más utilizado con los centros de salud. Tanto los ostomizados como sus familiares detectaban insuficiente asistencia $y$ coordinación entre atención primaria y atención hospitalaria.

Capacidad de autocuidado. Observamos en nuestra revisión, que prácticamente todos los pacientes ostomizados, eran autónomos para la realización de sus autocuidados. Un reducido 
grupo conseguía la autonomía en el hospital y la mayoría de los pacientes fuera de él.

Valores culturales-religiosos. Los musulmanes para acudir a sus oraciones deben realizar una purificación de las zonas sucias, en su cuerpo hay zonas limpias (por encima del ombligo) y zonas sucias (por debajo de éste). La presencia de un estoma en esta zona puede hacer que el musulmán creyente se sienta mal. Es importante que la educación sanitaria no interfiera con sus periodos de oración. Siempre que sea posible se debe colocar el estoma por encima del ombligo (práctica poco habitual) para evitar sentimientos negativos que poco o nada van a favorecer la recuperación y la adaptación.

En algunas comunidades árabes la manipulación del material sucio debe realizarse con la mano izquierda, esto puede complicar los cuidados hasta el punto de crear dependencia.

Los hindúes consideran prioritaria la limpieza del cuerpo para sus funciones religiosas y para la preparación de los alimentos. El estoma interfiere en sus condiciones de vida y en ocasiones puede producir el rechazo de la pareja. A menudo son vegetarianos, es importante valorar su estado nutricional y sus posibles carencias.

El judío cree en la perfección del cuerpo y en la ausencia de mutilaciones hasta la muerte. El estoma es para ellos, una mutilación difícil de olvidar, ya que tienen que realizar sus cuidados a diario.

Para los adventistas del séptimo día impera la sencillez en la vida, tienen prohibidos los narcóticos y los estimulantes. El aspecto más delicado es el relacionado con el dolor, nuestra actuación irá encaminada a informarles sobre los analgésicos sin forzar la situación, es importante que estemos atentos a sus manifestaciones, sobre todo las no verbales.

Los baptistas ven la enfermedad como un castigo divino. Su profunda creencia en la curación espiritual les hace reacios a menudo a los tratamientos médicos. Es preciso respetar sus convicciones intentando hacerles entender las ventajas del tratamiento y los peligros de no aceptarlo.

Para los budistas la prueba y el dolor son los forjadores del espíritu. Son estrictamente vegetarianos, estoicos ante el dolor y prefieren estar lúcidos ante la muerte.

\section{DISCUSIÓN}

La realidad después de la realización de un estoma, puede ser más desalentadora de lo que la mente había previsto.

Rev. Española de Enfermedades Digestivas. Varios autores.

La salud entendida como estado de bienestar físico, psíquico, social y espiritual, debe guiar al profesional enfermero a dirigir el proceso de cuidados, respetando los valores y creencias de las personas, su forma de vida y su cultura. En esta línea la profesión enfermera "es una profesión de cuidados transculturales, centrados en el cuidado humano, respetuosos de los valores culturales y del estilo de vida de las personas" (Luna y Cameron).

Es necesario subrayar que la cirugía traumática y mutilante, independientemente de lo esencial que sea, debe permitir a la persona vivir de forma normal lo antes posible. No se es suficientemente consciente de que las grandes intervenciones oncológicas en las que se realiza una derivación externa, se acompañan necesariamente de un gran impacto psicoemocional, que se traduce en severos problemas de personalidad.

La existencia de un estoma conlleva para el paciente:

- Una agresión muy importante a su imagen corporal.

- Una amenaza a la capacidad de autocontrol del individuo.

- Un elemento de incertidumbre en todas las esferas de su actividad.

- Una fuente continua e inagotable de angustia y depresión.

En el caso que nos ocupa, las personas ostomizadas, los factores educacionales, psicológicos, sociales y culturales influyen en el proceso de recuperación-adaptación al nuevo estado. Esta incidencia, en principio negativa (según los datos obtenidos), podría positivizarse con una educación individualizada que potencie hábitos saludables, con conocimientos sobre la situación y la vivencia personal-familiar de cada ostomizado y con una actuación interdisciplinar, basada sobre todo en la prevención de alteraciones. 
La función fisiológica de la evacuación intestinal, tiene una estrecha relación con la formación de la personalidad del individuo. La continencia es a la vez un símbolo de integración sociocultural y una señal diferenciadora del comportamiento animal; la pérdida de la continencia se vive como una alteración de la personalidad más grave que en el caso de otras mutilaciones, aunque éstas sean menos fáciles de disimular/ocultar que el estoma. Nuestra atención va a dirigirse principalmente a los trastornos psicosomáticos originados por la presencia del estoma.

El ostomizado se encuentra sujeto al estoma, por lo tanto precisa de una adecuada información y un adiestramiento, tanto práctico como teórico, que le permita desenvolverse con conocimiento y soltura en el manejo de su ostomía; no sólo a nivel físico o técnico, sino readaptándose a su nueva imagen corporal y vida social. En un porcentaje elevado los problemas psicológicos y sociales van a disminuir o solventarse a medida que la persona recupere la autoconfianza y la autoestima.

En nuestro medio, se da con frecuencia el hecho de que convivan diversas culturas, y los servicios sanitarios se dirigen en este momento a un mosaico cultural en donde se mezclan razas, nacionalidades, valores e idiomas diferentes. La comparación de modelos culturales ha de permitirnos identificar y explicar las claves en la transmisión cultural implicada en los procesos de salud. Si no actuamos según esta premisa antropológica, las diferencias culturales pueden actuar como filtros e interferir en la comunicación entre el docente y el usuario.

Conseguir empatizar con la persona, el respeto, saber escuchar, y mucho tacto son siempre ventajas importantes. Es necesario evitar toda actitud que pueda implicar un juicio moral y comportamientos capaces de constituir una amenaza para su autoestima.

En un estudio realizado a profesionales de enfermería, en la provincia de Tarragona, destacaron que un $28,1 \%$ tenía una actitud "negativa" hacia pacientes de etnias minoritarias. La actitud es la predisposición a actuar de una determinada manera, e incide de forma más o menos consciente en el comportamiento profesional.

Las personas procedemos de los más variados estratos sociales, culturales y religiosos y hemos sido condicionadas desde la infancia para aceptar diversas creencias, formas de vida y actitudes que dan lugar a una combinación única en cada individuo. El conocimiento del medio cultural y religioso de los pacientes y de su familia es importante ya que nos ayuda a individualizar el tratamiento y la información.

Si uno desea conocer lo que cabe esperar de un hombre, es necesario saber en que cultura fue educado y no a que raza pertenece.

"Cultura es el conjunto de los comportamientos, pensamientos y sentimientos implicados en el proceso de satisfacción de necesidades de un grupo humano" (Siles, 2.001)

D. Colom nos dice que aunque no es fácil, se debe trabajar el potencial existente en la familia y en el enfermo desde todas las perspectivas posibles: biológica, psicológica y sociocultural. En ocasiones la enfermedad representa una sobredosis de estrés, que en condiciones normales se superaría, pero que en un clima negativo y hostil significaría un agravamiento de las relaciones, que de ninguna forma garantizaría los cuidados.

La persona que pasa por la experiencia de una modificación importante de sus perspectivas de vida después de un problema de salud, encuentra con frecuencia un consuelo significativo en la presencia de sus allegados. Cuando se encuentra con ciertas limitaciones, ante la necesidad de proseguir su tratamiento o modificar hábitos de vida, es muy conveniente que pueda contar con la ayuda de sus parientes, amigos y/o vecinos, para reforzar su motivación y aumentar su perseverancia y fidelidad ante los imperativos a los que se encuentra sometida.

\section{CONCLUSIONES}

"El cuidado es el producto de la reflexión sobre las ideas, hechos y circunstancias, relacionadas con el proceso optimizador de las necesidades de salud que garantizan la integridad y la armonía de todas y cada una de las etapas que constituyen la vida humana" (Siles, 1.996, 2.000)

De la reflexión teórica, práctica y situacional, sobre el cuidado a los pacientes ostomizados, se evidencia la necesidad de una actuación profesional individualizada, las enfermeras deben ayudar a 
las personas para que aprendan actividades, mediante el proceso de enseñanza aprendizaje, dirigidas a satisfacer sus necesidades y a vivir con los efectos de los cuadros y estados patológicos, en un estilo de vida que favorezca el desarrollo personal continuado.

Como requisito previo a cualquier actividad debemos conocer la situación y las características específicas del paciente, de su familia y de su entorno, esto se conseguirá realizando una valoración integral. El éxito de nuestra actividad va a estar condicionado por la confianza, la competencia profesional y la disposición de aceptar a la persona que solicita ayuda. El proceso de atención va a estar basado y mediatizado por el tipo de relación que establezcamos y nuestras habilidades comunicativas, todo ello va a influir directamente en la recuperación/adaptación.

Concretando en la persona ostomizada, Nancy M. Holloway nos orienta sobre la valoración que deberíamos realizar. Nos centraremos en los patrones relacionados con el tema que nos ocupa:

\section{Patrón de percepción y mantenimiento de la} salud: explorar la percepción del paciente respecto a la ostomía y su impacto sobre su estado de salud y su estilo de vida.

Patrón de autoimagen y autoconcepto: el autoconcepto y la autoestima se correlacionan con el potencial de adaptación; estar atento a afirmaciones consistentes de autodesprecio o afectos inapropiados, que puedan indicar una baja autoestima. La respuesta emocional es variable, pero es común que los pacientes tengan sentimientos negativos respecto a la ostomía. La sinceridad en la expresión de los sentimientos se ve afectada por la personalidad del paciente y las capacidades de comunicación de la enfermera.

Patrón de rol y relaciones: valorar áreas de preocupación sobre roles y relaciones. Los pacientes generalmente expresan preocupación por el impacto de la ostomía en sus relaciones, con especial hincapié en la relación de pareja. Los adultos jóvenes y de edad media generalmente expresan preocupación sobre su capacidad para reanudar los roles y responsabilidades preoperatorios, mientras que los ancianos expresan preocupación por su capacidad para mantener la independencia y afrontar el costo de los materiales. Valorar la dinámica familiar, en particular los roles de dependencia/ independencia. Los pacientes mayores pueden desear implicar a su cónyuge u otras personas en su cuidado, mientras que los adultos jóvenes pueden valorar más la independencia e intimidad.

Patrón de sexualidad y reproducción: valorar la sinceridad del paciente y pareja entre sí y en la discusión sobre la sexualidad, patrones sexuales preoperatorios y otras preocupaciones importantes. Una preocupación común es el impacto de la ostomía sobre las relaciones íntimas; esto es, el atractivo y la función sexual.

Patrón de tolerancia al estrés: Las respuestas del paciente y familia a la ostomía son muy variables y reflejan sus patrones de adaptación. Valorar los sentimientos del paciente sobre los grupos de apoyo, para determinar la conveniencia de ponerle en contacto con alguna sociedad de ostomizados.

Patrón de valores y creencias: la respuesta a la ostomía se ve afectada por creencias culturales y la respuesta familiar a la enfermedad, cirugía y eliminación.

Tras conocer la situación del paciente, analizar y relacionar la información, llegaremos a una conclusión, a un diagnóstico sobre la respuesta del ostomizado a su problema de salud. Es importante comprobar con él los diagnósticos y priorizarlos según su importancia. Posteriormente, y siempre con el protagonista de la situación, marcaremos unos objetivos, y unos plazos para alcanzarlos y solventar los problemas, junto con las actividades necesarias para conseguirlo. De forma continuada y al finalizar los plazos marcados evaluaremos los resultados obtenidos.

Teniendo en cuenta las diferentes alteraciones que puede presentar el ostomizado, tanto Nancy M. Holloway como L.J. Carpenito, exponen en los Planes de Cuidados Estandarizados, los Diagnósticos de Enfermería comunes en estas personas, quedando la adaptación para el propio ejercicio según las características individuales de cada 
persona. Los más relacionados con el tema de este trabajo, son los siguientes:

- Ansiedad debida a la falta de conocimientos acerca de los cuidados de la ostomía y a los efectos negativos percibidos en el estilo de vida.

- Posible alteración del autoconcepto debido a los efectos de la ostomía sobre la imagen corporal y el estilo de vida.

- Posible alteración de los patrones de sexualidad debido a la percepción del impacto negativo de la ostomía sobre la actividad y el atractivo sexual.

- Posible disfunción sexual debida a la impotencia fisiológica, secundaria a una lesión del nervio simpático (varón) o a una inadecuada lubricación vaginal (mujer).

- Posible aislamiento social debido a la ansiedad ante el posible olor y fuga del equipo.

- Deterioro de la comunicación verbal en relación con incapacidad de producir el habla secundario a traqueostomía.

- Potencial alteración de los patrones sexuales en relación con el cambio de aspecto, miedo al rechazo.

Una vez definida la situación específica, el objetivo principal de nuestra actuación va a ser la enseñanza de conductas positivas, dirigidas a optimizar los mecanismos de satisfacción de sus necesidades, así como autonomía e independencia. Esto lo conseguiremos mediante la educación sanitaria, herramienta clave de toda nuestra actuación. Con ella vamos a dotar a la persona de los conocimientos, actitudes y habilidades necesarias para que participe activamente en el tratamiento de su enfermedad o de las secuelas de ésta.

Hacemos nuestras las palabras de D. Colom, que nos señala 12 aspectos a tener en cuenta por el equipo interdisciplinar, ante procesos crónicos, para organizar la intervención:

1. Tratar las pérdidas que la enfermedad implica a medio y largo plazo.

2. Garantizar el máximo soporte por parte del equipo que lo trata y facilitar su accesibilidad.

3. Identificar a la persona de referencia según el enfermo. Ésta no tiene porqué coincidir con la persona que asume las directrices de la familia.

4. Facilitar elementos de seguridad al cuidador/a. Muchas veces éste es el que menos soporte recibe por parte de los profesionales y del resto de la familia, y se le exige más de lo que puede dar.

5. Promover grupos de soporte o ayuda mutua, en los que los protagonistas son los miembros responsables de dar la atención.

6. Ayudar al enfermo y a su familia a intelectualizar el proceso de la enfermedad y deterioro.

7. No banalizar o exagerar la importancia que tiene la enfermedad.

8. Dejar que la persona exprese angustias y temores. Si éstos superan las posibilidades de atención del profesional, cabe recurrir a especialistas.

9. Sensibilizar a los miembros de la red social del enfermo para conseguir su colaboración sin sobrecargar a las personas cuidadoras, puesto que de su colaboración depende que no se demande a la institucionalización.

10. Mantener las relaciones con el entorno tanto en frecuencia como en calidad.

11. Fomentar actividades sanas: deportes, paseos, alimentación...

12. Estudiar las condiciones de la vivienda y valorar si el empeoramiento puede significar un incremento de las barreras arquitectónicas.

Podemos concluir afirmando que los ostomizados sufren a lo largo de su vida un continuo proceso de adaptación consistente en adecuar su vida al nuevo elemento, llegando difícilmente a controlarlo por el desconocimiento sobre el tema que poseen.

La persona deberá aprender a vivir con el estoma, debe reorganizar su imagen corporal, adaptarse a lo que la pérdida de continencia conlleva (fugas, olores, gases...), solventar la incidencia del estoma y/o del tipo de intervención en su vida sexual, y volver a la dinámica familiar, social y laboral. El adiestramiento debe ser realizado por personal preparado, planificado desde el momento del diagnóstico y prolongarse hasta después del alta.

\section{El Plan Terapéutico ira encaminado a:}

- Proporcionar apoyo mediante una intercomunicación eficaz.

- Dar información clara y repetida, sin miedos, con el tiempo necesario posibilitando al paciente que despeje dudas, temores infundados, confusiones, etc.

- Conseguir por parte del paciente un dominio activo de la situación planteada como consecuen- 
cia de la ostomía, procurando que no asuma mecanismos de defensa inapropiados como el mecanismo de negación prolongado.

- Cultura, Educación y Cuidados, han sido los ejes sobre los que hemos ido desarrollando el presente trabajo, inseparables e interrelacionados entre sí, nos han demostrado la utilidad de la Antropología Educativa de los Cuidados, para realizar un proceso de transmisión cultural que aporte estilos de vida saludables a los pacientes ostomizados, mediante las herramientas básicas de la Antroplogía, la Educación y la Enfermería.

\section{BIBLIOGRAFÍA}

ARAGONÉS, AM.; SEGURA, A.; BILBAO, MC.; RUBIO, JS.; JUSTE, JF. (1991). La sexualidad y la información en los pacientes ostomizados: nuestra experiencia en 23 casos. Enfermería Científica 111, 23-27.

BRECKMAN, B. (1990). Enfermería del estoma. Edición especial para Convatec, Madrid pp. 1112.

CAMPBELL, C. (1987). Tratado de Enfermería. Diagnósticos y Métodos. Doyma, Barcelona.

CARPENITO, LJ. (1994). Planes de Cuidados y Documentación en Enfermería. En Diagnósticos de Enfermería y problemas asociados. Interamericana McGraw-Hill, Madrid pp. 419474 y $553-689$.

COLLIÉRE, M. (1989). Utilización de la antropología para abordar situaciones de cuidados. Rol de Enfermería, 179-180: 17-25

COLOM, D. (1993). La intervención multidisciplinar de los equipos de salud y su influencia en la corresponsabilidad familiar y social. Enfermería Clínica, 3(4), 4-7.

FLOREZ LOZANO, JA. (1994). Aspectos psicológicos del paciente ostomizado. Edición especial Coloplast productos médicos S.A.

HENDERSON, VA. (1994). La naturaleza de la enfermería. Reflexiones 25 años después. Interamericana Mcgraw-Hill. Madrid.

HERNAN SAN MARTíN. (1979). Ecología humana y salud. La Prensa Médica Mexicana, 132. México.

HOLLOWAY, NM. (1990). Planes de cuidados en Enfermería Médico-Quirúrgica. Editorial Doyma, Barcelona pp. 238-364.

LUNA Y CAMERON. (1996). En: Kèrouac. "El pensamiento enfermero". Masson, Barcelona pp. 45
MARRINER-TOMEY (1994). Modelos y teorías de Enfermería. Mosby-Doyma libros. Madrid.

MARTÍNEZ, MC.; MERCADER, J.; HERNÁNDEZ J, OLMOS L. (1990). La sexualidad del paciente ostomizado. Española de Enfermedades Digestivas; Oct.1990, 210-214.

MARTOS MONEREO, MA.; ESPINOSA ARIETE D.; GARRIDO HIGUERAS V. (1993). Programa de educación para la salud mediante visita domiciliaria al enterostomizado y familia. Enfermería Científica 139, 4-7.

MARTOS MONEREO, MA. (1992). Justificación para la formación continuada en ostomías. Enfermería Científica 126, 11-14.

PARDO SERRANO, C.; MALLEBRERA CAÑADA, E.; REOLID COLLADO, M.; LOPEZ CAMPILLO, R.; COLA PALAO, A. (1992). Judaísmo y Salud. Enfermería Científica 129, 47.

PETERS-GAWLIK, M. (1997). Un modelo teórico para el asesoramiento psicosocial en estomaterapia. Rev. Del CORCE. Euroestoma 18, 12-13.

PÉREZ GARCÍA, S. (1991). Educación sanitaria: medio para mejorar la salud del ostomizado. Enfermería Científica 117, 4-11.

PÉREZ, S.; BARBERO, MA.; CAMPO, J. (1993). Estudio comparativo sobre el impacto de un programa de Educación Sanitaria en la persona urostomizada. Enfermería Científica 138, 4-18.

RENTERO, A.; SANTJUSTE, M.; SALORIO, P.; FLORES, MD. (1993). Protocolo de información al paciente ostomizado para enfermería. Enfermería Científica 132, 4-8.

RIPOLlE, L.; GRONDIN, L.; PHANEUF, M. (1993) Cuidados de Enfermería. En Aplicación del proceso enseñanza-aprendizaje. Interamericana-McGraw-Hill, Madrid cap. 5: 132.

RODRÍGUEZ, J.; LÓPEZ, S.; GARCÍA, J. et al. (1998). Problemática psicosocial del paciente ostomizado. Rol de Enfermería 114, 21-23.

ROVIRA VECIANA, MA.; URIZ SOLÁ, E.; RODRÍGUEZ SUAREZ, C.; VILÁ CORCOLES, A.. (1993). Actitud de los profesionales de enfermería ante los pacientes pertenecientes a etnias minoritarias. Enfermería Clínica, 3(1), 1620.

SILES et al. (1997). Por una rentabilización pedagógica en la obra de Benedetti: etnología narrativa y situaciones de vida-salud. Cultura de los Cuidados 1, 17-24

VAQUER CASAS, G.; GIRALS RUBIROLA, M. (1992). Enfermería y Estomaterapia. Enfermería Científica 120, 7-18. 